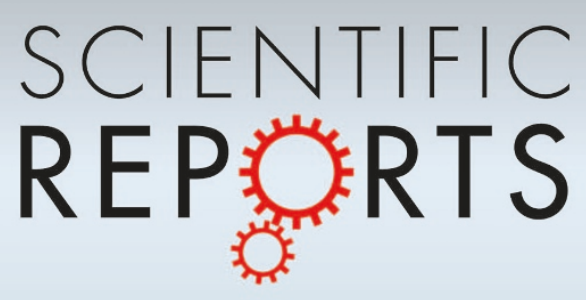

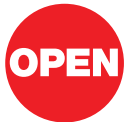

SUBJECT AREAS:

NANOWIRES

SURFACES, INTERFACES AND

THIN FILMS

ELECTRONIC PROPERTIES AND

DEVICES

SCALING LAWS

Received

3 January 2013

Accepted

7 March 2013

Published

22 March 2013

Correspondence and requests for materials should be addressed to Q.J. (jiangq@jlu.edu.

$\mathrm{cn})$

\section{Physicochemical insight into gap openings in graphene}

\author{
Y. F. Zhu, Q. Q. Dai, M. Zhao \& Q. Jiang
}

Key Laboratory of Automobile Materials (Jilin University), Ministry of Education, and School of Materials Science and Engineering, Jilin University, Changchun, 130022, China.

Based on a newly developed size-dependent cohesive energy formula for two-dimensional materials, a unified theoretical model was established to illustrate the gap openings in disordered graphene flakes, involving quantum dots, nanoribbons and nanoporous sheets. It tells us that the openings are essentially dominated by the variation in cohesive energy of $\mathrm{C}$ atoms, associated to the edge physicochemical nature regarding the coordination imperfection or the chemical bonding. In contrast to those ideal flakes, consequently, the gaps can be opened monotonously for disordered flakes on changing their sizes, affected by the dimension, geometric shape and the edge saturation. Using the density functional theory, accordingly, the electronic structures of disordered flakes differ to the ideal case because of the edge disorder. Our theoretical predictions have been validated by available experimental results, and provide us a distinct way for the quantitative modulation of bandgap in graphene for nanoelectronics.

$\mathrm{n}$ the fields of materials science, increasing interest is now being attracted to graphene, a two-dimensional (2D) sheet composing of carbon atoms arranged in a honeycomb lattice structure ${ }^{1}$. Because of its compatibility with industry-standard lithographic processing, electron mobility up to 150 times greater than Si and a thermal conductivity of graphene twice of that of diamond ${ }^{2}$, graphene shows its promise as a future material for the nanoand micro-electronics applications, such as field effect transistors (FETs) ${ }^{3,4}$, biological membranes ${ }^{5}$, sensitive platforms ${ }^{6,7}$, and so on. Among them, as the most successful device concept in electronics, most works on graphene devices so far have been related to $\mathrm{FETs}^{8,9}$. When graphene is used as the channel material, the graphene-based transistors will own an ultrafast speed, making it able to operate in the $\mathrm{THz}$ frequency range $\mathrm{e}^{10}$. Moreover, the extremely thin channels will possibly allow the transistors to be scaled to shorter channel lengths without encountering the adverse short channel effects, which restrict the performance of existing devices.

To achieve graphene-based electronic devices with high performance, specific bandgaps of graphene are usually required for different electronic applications ${ }^{11-13}$. As instance, since the $I_{\text {on }} / I_{\text {off }}$ ratios in FETs are largely dependent on $E_{\mathrm{g}}$ of the channel materials on the basis of their proportional relationship with $I_{\mathrm{on}} / I_{\mathrm{off}} \propto \mathrm{e}^{E_{g} / k_{\mathrm{B} T}}$, graphene materials with sufficiently large bandgaps are demanded for room-temperature FET operation ${ }^{14-16}$, where $E_{\mathrm{g}}$ denotes the bandgap, $k_{\mathrm{B}}$ the Boltzmann constant and $T$ the absolute temperature. In general, $E_{\mathrm{g}}$ with only a few tens of meV is too weak for room-temperature operation of FETs ${ }^{14}$. To have $I_{\text {on }} / I_{\text {off }}$ ratios as $10-100, E_{\mathrm{g}}$ of graphene materials around $0.1 \mathrm{eV}$ is required ${ }^{15}$, while for high $I_{\mathrm{on}} / I_{\text {off }}$ of $10^{7}$, it should be large as $0.4 \mathrm{eV}^{15,16}$. Despite this, however, graphene cannot be directly adopted as semiconductors in electronics because of its zero bandgap $\left[E_{\mathrm{g}}(\infty)=0 \mathrm{eV}\right]^{17}$, where $\infty$ denotes the bulk size in $2 \mathrm{D}$. With such an issue, graphene creates a unique set of challenges for implementation into conventional electronics due to substantial leakage currents in the off state for field-effect devices. To address it, the degeneracy of the conduction and valance bands should be lifted via breaking the equivalence between the $\mathrm{A}$ and $\mathrm{B}$ sublattices ${ }^{18}$. Aiming at this purpose, the bandgap openings (BOs) in graphene have been realized by forming graphene quantum dots (GQDs) ${ }^{2,19,20}$, nanoribbons (GNRs) , $15,21-23^{2}$ and nanoporous graphene sheets (NPGs) ${ }^{24,25}$.

Upon the investigation on the BOs in GQDs, GNRs or NPGs, different edge natures should be considered. According to its honeycomb lattice, graphene may own ideal armchair (AC) or zigzag (ZZ) edges ${ }^{12}$. With the present fabrication techniques, however, these perfect edges are difficult to be formed for GQDs and GNRs ${ }^{26,27}$. Instead, disordered edges are usually observed, which can be regarded as the mixture of AC and ZZ edge shapes $^{2,28}$. Because of it, the two edge structures of GNRs remain asymmetric ${ }^{2,26,27}$. Moreover, their edges are kept naked with one dangling bond for each $\mathrm{C}$ atom. To stabilize those $\mathrm{C}$ atoms, dangling bonds can be chemically saturated with some radicals or groups denoted by $\mathrm{R}$ beneficial for the $\mathrm{BO}$, such as $\mathrm{R}=\mathrm{H}^{29}, \mathrm{~F}^{30}$ or $\mathrm{OH}^{31}$. Such an edge saturation was not performed for GQDs and GNRs in most experiments due to its technical difficulty, while Ritter et al. ${ }^{2}$ claimed that it can be performed through exposing GQD or GNR flakes to H radicals. Disordered 
GQDs and GNRs without the edge saturation will be shown as GQDnull and GNR-null, while those saturated with $\mathrm{R}$ will be exhibited as GQD-R and GNR-R. As to mesoscopic NPGs ${ }^{24,25}$, the holes in graphene are usually fabricated by the lithography technique with different geometrical shapes, such as circles or squares (C-NPGs or SNPGs). Far from the atomistic precision, the internal edges are usually kept disordered and naked, referred to as DO-NPG-null. Owing to these attempts, the BOs were observed in disordered GQDs ${ }^{1,20}$, $\mathrm{GNRs}^{3,15,21,23,32-34}$ and NPGs ${ }^{24,25}$, while the $D$-dependence of $E_{\mathrm{g}}(D)$ varies considerably among them, where $D$ denotes the diameter of GQDs or holes in C-NPGs, width of GNRs, or the side length of holes in S-NPGs. On lowering their $D$, a monotone increase in the openings was observed for disordered and naked GQDs and $\mathrm{GNRs}^{3,15,19-21,23,32-34}$, while it is more substantial for GQDs. As regards NPGs, a monotone decrease in the BOs has been reported ${ }^{24}$. In contrast, the BOs are lowered when their edges are saturated ${ }^{2}$. However, our understanding to the openings in disordered flakes is still in incubation.

Much effort has been devoted to investigating the opening mechanism in graphene. As a powerful technique, the computer simulation approaches, such as the first principles density functional theory (FP-DFT), Dirac equation and the tight-binding (TB) approximations, have been adopted for such studies. It may provide us band structure images, electronic density and other basic information of graphene for analyzing and explaining the electronic performance ${ }^{35}$. By this means, the opening behavior is usually studied using ideal GQDs and GNRs with regular edge structures ${ }^{13,29,31,36,37}$ or ideal NPGs with perfect periodic antidot superlattices or hole ${ }^{38-40}$. With these contributions, however, it seems still difficult to illustrate the openings in disordered flakes, since these ideal flakes exhibit much different $D$-dependences of $E_{\mathrm{g}}(D)$. The BOs in ideal GQDs and GNRs are relevant to edge structures. AC-GQDs have larger $E_{\mathrm{g}}(D)$ than ZZGQDs $\mathrm{do}^{36}$. By exploring the electronic properties for a wide class of nanoribbons, GNRs exhibit a rich variety of bandgaps from metals to typical semiconductors ${ }^{13,37}$. Typically, ZZ-GNRs show a metallic or semiconducting state ${ }^{13,29,31,37}$, while AC-GNRs present the oscillating $\mathrm{BO}$ behaviors from the metallic to semiconducting state with respect to $D^{13,29,37}$, where the metallic points emerge at the period of 3 with $N_{\mathrm{a}}$ $=3 p+2, N_{\mathrm{a}}$ the number of AC chains and $p$ a positive integer. Concerning ideal NPGs $s^{38-40}$, the opening behaviors are much complicate, which may be affected by not only the hole diameter but also the hole geometry, lattice geometry, separation between adjacent holes, hole locations and the edge geometry. When $D$ of holes rises, $E_{\mathrm{g}}(D)$ would increase for some antidot lattices ${ }^{39}$, such as hexagonal or triangular antidot lattices with a hexagon-shaped hole, while for the rectangular, rotated triangular and the honeycomb antidot lattices with a hexagon-shaped hole, only particular hole separations lead to an obvious opening ${ }^{39}$. The rectangular antidot superlattices perforated with four holes of honeycomb symmetry can exhibits the semiconducting or metallic states, depending on the width ratio of AC chains to $\mathrm{ZZ}$ chains ${ }^{38}$. More impressively, in particular, ACGNRs with $N_{\mathrm{a}}=11$ can be changed from the metallic state to the semiconducting state when it is perforated with a honeycomb hole especially at the central position, while it is still metallic if a triangular-shape hole is introduced ${ }^{38}$.

Besides the above studies, some other theoretical ways have been explored for the BOs in DO-GNRs by considering the impact from the edge disorder ${ }^{31}$. With the renormalized lateral confinement approach ${ }^{41}, E_{\mathrm{g}}(D)$ of DO-GNRs is decided by the structural disorder at the edges caused by etching or inaccuracies in the width determination, while such a disorder can result in an "effective transport width" smaller than the ribbon's nominal width. Alternatively, the BO behaviors can be explained with the Coulomb blockade or charging effects ${ }^{22,26,27}$, where the BOs are induced not only by confinement but also by Coulomb blockade originated from the edge roughness. However, the BOs in disordered GQDs and NPGs have been less discussed. As shown in Table 1, in addition, some expressions have been presented to plot $E_{\mathrm{g}}(D)$ of disordered GQDs, GNRs or NPGs. In general, these expressions are achieved using the fitting method with those experimentally measured $E_{\mathrm{g}}(D)$ values, while one or two adjustable parameters $a$ and/or $b$ are present there. Since these parameters are case-dependent and vary considerably among different researches, the reported fitting expressions cannot take their essential roles in helping us revealing the opening mechanism either.

Due to the limitation in existing approaches or expressions, some issues on the BOs in disordered graphene flakes have not been addressed. As instances, a theoretical way to describe the difference in $E_{\mathrm{g}}(D)$ induced by the dimension effect is still absent. It also remains unclear how to depict the reverse change in $E_{\mathrm{g}}(D)$ of NPGs relative to those of GQDs and GNRs. As to the role from the edge saturation, on the other hand, its exact mechanism has not been clarified as yet. On account of it, one still wonders how the BOs in saturated flakes will be weakened relative to those naked ones. In practice, the gap opening mechanism of disordered flakes is much complicated, which is relevant to not only the size but the dimension, geometric shape and the edge saturation. However, a systematic theoretical investigation on their roles is still absent, which obstructs our physical insight into the BOs and throws obstacles in our way toward the full-scale application of graphene in electronics. Because of these, a unified theoretical approach should be developed to elucidate the BOs in disordered graphene flakes.

In recent years, with the use of the mesoscopic thermodynamics of materials ${ }^{42,43}$, a bandgap thermodynamics has been proposed to illustrate the $E_{\mathrm{g}}(D)$ function of $3 \mathrm{D}$ III-V and II-VI nanosemiconductors with macroscopic parameters of materials based on the nearly-freeelectron approach ${ }^{44-46}$. In light of it, strikingly, it reads,

$$
E_{g}(D)=\left[2-E_{c}(D)_{3 D} / E_{C}(\infty)\right] E_{g}(\infty) .
$$

In equation 1.1, $E_{\mathrm{c}}(D)_{3 \mathrm{D}}$ is the energy required to break the atoms in the solid into isolated atomic species, which can be obtained experimentally by measuring the latent heat of sublimation at any temperature, and extrapolating to zero Kelvin. Inspired by Lindemann's criterion for solid melting and Mott's expression for

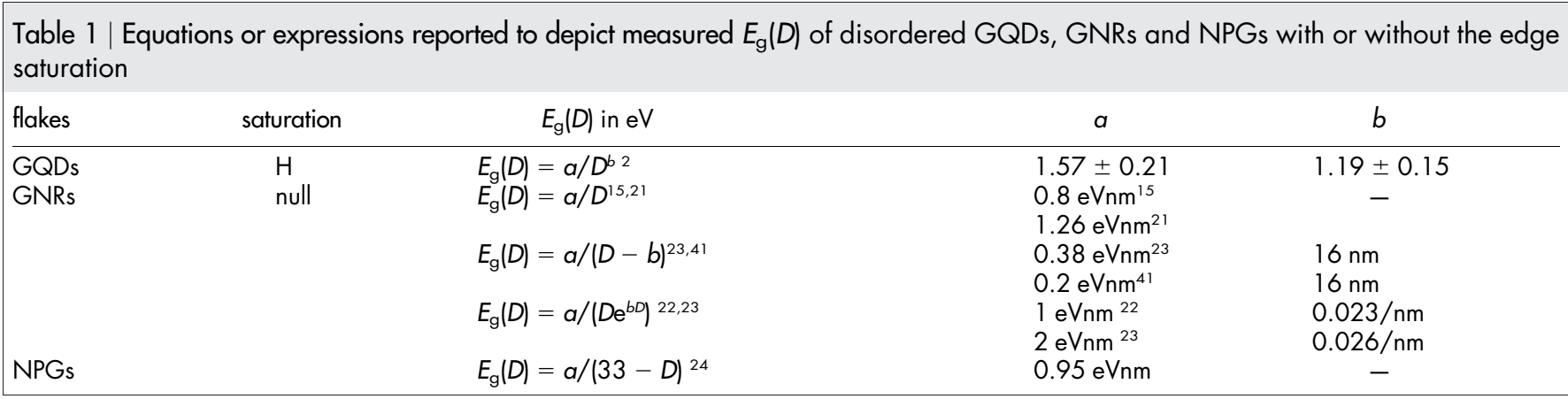


vibrational melting entropy ${ }^{47-50}$, utilizing the proportional relationship between $E_{\mathrm{c}}(D)$ and $T_{\mathrm{m}}(D), E_{\mathrm{c}}(D)_{3 \mathrm{D}} / E_{\mathrm{c}}(\infty)$ function for the $3 \mathrm{D}$ nanocrystals having free surfaces with broken bonds is given as ${ }^{44,45}$,

$$
E_{c}(D)_{3 \mathrm{D}} / E_{c}(\infty)=\exp \left[-\left(\alpha_{3 \mathrm{D}}^{0}-1\right) /\left(D / D_{3 \mathrm{D}}^{0}-1\right)\right],
$$

where $\alpha_{3 \mathrm{D}}^{0}$ is a surface physicochemical amount with $\alpha_{3 \mathrm{D}}^{0}=$ $\sigma_{\text {sv }}(D)^{2} / \sigma_{\text {in-3D }}(D)^{2}, \sigma^{2}$ the mean square displacement of thermal vibration at the melting temperature, and the subscripts $s v$ and in$3 D$ the surface and interior atoms. Using Lindemann's criterion for solid melting and Mott's expression ${ }^{49,50}$, it was derived that $\alpha_{3 \mathrm{D}}^{0}=$ $2 S_{\text {vib }}(\infty) / 3 R+1$, where $S_{\text {vib }}(\infty)$ denotes the atomic vibration entropy and $R$ the gas constant. $D / D_{3 \mathrm{D}}^{0}=v / s h$ or $D_{3 \mathrm{D}}^{0}=2(3-d) h$ denotes the critical diameter of crystals where almost all atoms or molecules are located on the surface and a crystalline structure is no longer stable, $v / s$ means the volume/surface ratio, $d$ the dimension of the crystal with $d=0$ for nanoparticles, $d=1$ for nanowires and $d=2$ for thin films. In light of equations 1.1 and 1.2, the bandgap expansion for nanoscaled 3D III-V and II-VI semiconductors induced by the surface or interface effect has been illustrated successfully ${ }^{44-46}$. Stimulated by this success, one may conceive that the aforesaid issues on the $\mathrm{BOs}$ in the $2 \mathrm{D}$ graphene system may also be addressed in the thermodynamic way. Figure 1 illustrates the schematic plots of (A) GQDs, (B) GNRs, (C) C-NPGs and (E) S-NPGs, while a tripolar flake marked with $\mathrm{YA}_{1} \mathrm{~A}_{2} \mathrm{~B}_{1} \mathrm{~B}_{2} \mathrm{C}_{1} \mathrm{C}_{2}$ in (D) and a cross flake with $+\mathrm{A}_{1} \mathrm{~A}_{2} \mathrm{~B}_{1} \mathrm{~B}_{2} \mathrm{C}_{1} \mathrm{C}_{2} \mathrm{D}_{1} \mathrm{D}_{2}$ in (F) denote the nanoscopic functional units of C-NPGs in (C) and S-NPGs in (E), respectively. In view of it, an atomic layer can be observed at the edge of GQDs, GNRs and tripolar or cross flakes, which should be responsible for the BOs in graphene. With this way, the distinct edge physicochemical nature of $\mathrm{C}$ atoms, such as coordination imperfection (CI) or chemical bonding, should play an important role in the BOs in graphene with relation to the dimension, geometric shape and the edge saturation. However, no attempts have been made on it so far.

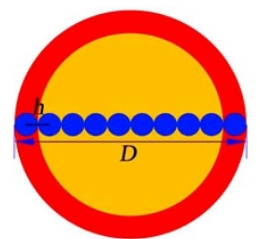

(A) GQDs

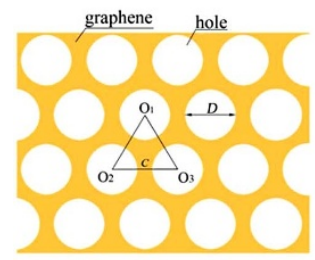

(C) C-NPGs

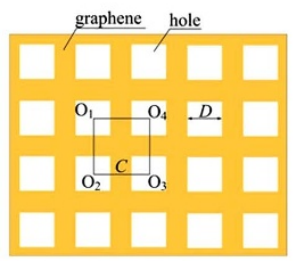

(E) S-NPGs

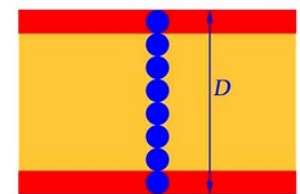

(B) GNRs

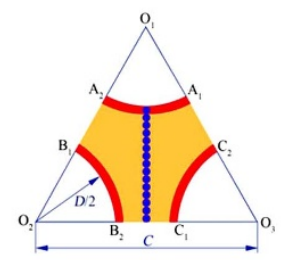

(D) tripolar unit of (C)

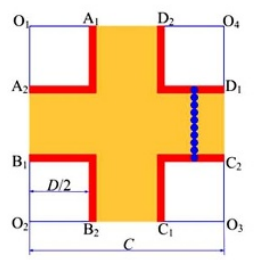

(F) cross unit of (E)
Figure 1 Illustration of edge atomic layers (marked in red) for (A) GQDs, (B) GNRs, (C) C-NPGs, (D) tripolar unit of (C), (E) S-NPGs, and (F) cross unit of $(\mathrm{E})$.
In this paper, a general thermodynamic approach will be proposed to illustrate the BO behaviors in disordered graphene flakes. Based on this, significantly, it provides us a new physicochemical insight into the openings in graphene. With the DFT simulation, accordingly, the electronic structure of disordered GNRs will be investigated. Suggestions will be made how to tune $E_{\mathrm{g}}(D)$ of graphene for its application in nanoelectronics.

\section{Results}

BOs in disordered and naked GQDs, GNRs and NPGs. In light of equations 2.1 and 2.2 in methods, $E_{\mathrm{g}}(D)$ of naked graphene flakes can be worked out as long as $\alpha_{2 \mathrm{D}}^{0}$ and $D_{2 \mathrm{D}}^{0}$ are known. Since $\alpha_{3 \mathrm{D}}^{0}$ and $\alpha_{2 \mathrm{D}}^{0}$ are decided by the CI of atoms at the surface of 3D nanocrystals or edge of $2 \mathrm{D}$ graphene flakes, $\alpha_{2 \mathrm{D}}^{0}$ can be correlated with $\alpha_{3 \mathrm{D}}^{0}$ via the coordination number $C N$ of atoms at the surface $N_{\text {sv }}$ and that at the edge $N_{\text {edge. }}$ According to the inverse proportion relationship between $\sigma^{2}$ and $C N$ of atoms, based on the definitions of $\alpha_{3 \mathrm{D}}^{0}$ in Introduction and $\alpha_{2 \mathrm{D}}^{0}$ in Methods, one has $\alpha_{3 \mathrm{D}}^{0} \propto\left(N_{\mathrm{sv}} / N_{\mathrm{in}-3 \mathrm{D}}\right)^{-1}$ and $\alpha_{2 \mathrm{D}}^{0} \propto$ $\left(N_{\text {edge }} / N_{\text {in-2D }}\right)^{-1}$, where $N_{\text {in-3D }}$ and $N_{\text {in-2D }}$ denote the $C N$ in the bulk $3 \mathrm{D}$ and $2 \mathrm{D}$ crystals. It thus reads $\alpha_{2 \mathrm{D}}^{0} / \alpha_{3 \mathrm{D}}^{0}=\left(N_{\mathrm{sv}} / N_{\mathrm{in}-3 \mathrm{D}}\right) /$ $\left(N_{\text {edge }} / N_{\text {in-2D }}\right)$. Since $N_{\text {in-3D }}=12$ and $N_{\text {sv }}=9$ for face-centered cubic crystals and $N_{\text {in }-3 \mathrm{D}}=8$ and $N_{\mathrm{sv}}=6$ for body-centered cubic ones $^{47}, N_{\text {sv }} / N_{\text {in }-3 \mathrm{D}}=3 / 4$. Moreover, $N_{\text {edge }}=2$ and $N_{\text {in }-2 \mathrm{D}}=3$ for $2 \mathrm{D}$ graphene flakes. On the basis of these, $\alpha_{2 \mathrm{D}}^{0}$ can therefore be elucidated with $\alpha_{2 \mathrm{D}}^{0}=9 \alpha_{3 \mathrm{D}}^{0} / 8$. Utilizing the published $\alpha_{3 \mathrm{D}}^{0}$ expression cited in Introduction, one has $\alpha_{2 \mathrm{D}}^{0}=9\left[2 S_{\mathrm{vib}}(\infty) / 3 R+1\right] / 8$.

As regards $D_{2 \mathrm{D}}^{0}$ of GQDs, GNRs and NPGs, it can be given with equation 2.4. Since $l / s=2(2-d) / D, D_{2 \mathrm{D}}^{0}$ of GQDs and GNRs can be given as $D / D_{2 \mathrm{D}}^{0}=D /[2(2-d) h]$ or $D_{2 \mathrm{D}}^{0}=2(2-d) h$, where $d=0$ for GQDs and $d=1$ for GNRs. As regards NPGs, $D_{2 \mathrm{D}}^{0}$ cannot be decided directly with that for GQDs or GNRs. This is because $D$ here denotes not the size of functional tripolar or cross flake but that of holes in NPGs. Given that the effective size of tripolar or cross flakes is $D_{\text {eff }}$, $D_{\text {eff }} / D_{2 \mathrm{D}}^{0}$ should be explored using $D_{\text {eff }} / D_{2 \mathrm{D}}^{0}=s /$ lh. For the tripolar unit of C-NPGs in Figure 1(D), the edge-C atoms along the concave edges of $A_{1} A_{2}, B_{1} B_{2}$ and $C_{1} C_{2}$ own broken bonds, while those along straight lines of $\mathrm{A}_{2} \mathrm{~B}_{1}, \mathrm{~B}_{2} \mathrm{C}_{1}$ and $\mathrm{C}_{2} \mathrm{~A}_{1}$ are still embedded in graphene without any $\mathrm{CI}$, where not the latter but the former takes effect in the BOs of NPGs. Hence, the effective free edge $l$ of $Y A_{1} A_{2} B_{1} B_{2} C_{1} C_{2}$ comes from three concave sections of $A_{1} A_{2}, B_{1} B_{2}$ and $C_{1} C_{2}$ only. Let the distance between the center of any two neighboring circles $\mathrm{O}_{1}, \mathrm{O}_{2}$ and $\mathrm{O}_{3}$ be $C$ larger than $D, l=\mathrm{A}_{1} \mathrm{~A}_{2}+\mathrm{B}_{1} \mathrm{~B}_{2}+\mathrm{C}_{1} \mathrm{C}_{2}=3 \mathrm{~A}_{1} \mathrm{~A}_{2}=\pi D /$ 2 , and $s=\sqrt{3} C^{2} / 4-\pi D^{2} / 8$. Thus, we have $l / s=4 \pi D /\left(2 \sqrt{3} C^{2}-\right.$ $\left.\pi D^{2}\right)$. Taking it into the above $D_{\text {eff }} / D_{2 \mathrm{D}}^{0}$ expression, one has $D_{\text {eff }} / D_{2 \mathrm{D}}^{0}$ $=\left(2 \sqrt{3} C^{2}-\pi D^{2}\right) /(4 \pi D h)$. Concerning the cross unit of S-NPGs in Figure $1(\mathrm{~F})$, in analogy, $l / s=4 D /\left(C^{2}-D^{2}\right)$, and $D_{\text {eff }} / D_{2 \mathrm{D}}^{0}=\left(C^{2}-\right.$ $\left.D^{2}\right) /(4 D h)$. Inserting these $D_{\text {eff }} / D_{2 \mathrm{D}}^{0}$ expressions into equation 2.2 instead of $D / D_{2 \mathrm{D}}^{0}, E_{\mathrm{g}}(D)$ as the function of $D$ can be explored for C- and S-NPGs.

With the above amounts, Figure 2 shows the solid $E_{\mathrm{g}}(D)$ curves as the function of $D$ for (A) GQD-null or GNR-null, (B) C-NPG-null and (C) S-NPG-null plotted with equations 2.1 and 2.2. For the comparison purpose, the reported reciprocal or exponential fitting expressions are also shown using dashed and/or dotted curves for GNR-null ${ }^{15,41,21,23}$ and NPG-null ${ }^{24}$. Available experimental results (symbols) were shown accordingly for verification. In light of Figure 2, the bandgaps are substantially opened for naked GQDs, GNRs and NPGs. As $D$ shrinks, $E_{\mathrm{g}}(D)$ of GQDs and GNRs is expanded exponentially. Moreover, GQDs have larger $E_{\mathrm{g}}(D)$ values than GNRs do. In contrast, much similar $D$-dependence of $E_{\mathrm{g}}(D)$ has been observed for C-NPGs in (B) and S-NPGs in (C), where $E_{\mathrm{g}}(D)$ shrinks on decreasing $D$, and $E_{\mathrm{g}}(D)$ becomes strong as $C$ declines from $44 \mathrm{~nm}$ to $22 \mathrm{~nm}$. Our predicted curves go through experimental results shown in Figure 2(A)-(B), supporting that our theoretical approach can be utilized to elucidate the BOs in graphene regarding the dimension and geometrical shape. As regards the upward or 
downward deviation of reported $E_{\mathrm{g}}(D)$ values of GNRs from our predictions, it should be attributed to the methods to measure $E_{\mathrm{g}}(D)$. As exampled by GNRs, in particular, its $E_{\mathrm{g}}(D)$ is usually derived from the $I_{\mathrm{on}} / I_{\text {off }}$ ratios of GNR-based FETs ${ }^{3,15,16,51}$. Utilizing this means, there will be some possible errors caused by inaccurate $D$ determination due to over-etching underneath the etch mask, uncertainties in the $D$ measurements based on atomic form microscope or the assumption of negligible Schottky barrier for holes in ultra-narrow GNR-based FETs ${ }^{15,41}$.

BOs in disordered but saturated GQDs and GNRs. Using equations 2.1 and $2.2, E_{\mathrm{g}}(D)$ of saturated GQDs and GNRs can be explored if $\alpha_{2 \mathrm{D}}^{\mathrm{R}}$ is known, where $D_{2 \mathrm{D}}^{0}$ is the same as that of naked GQDs and GNRs. Since $\alpha_{2 D}$ is related to the edge atomic nature, $\alpha_{2 D}^{R}$ may be resolved via $\alpha_{2 \mathrm{D}}^{0}$. According to equation 2.3 , it reads $\alpha_{2 \mathrm{D}}^{\mathrm{R}}=$ $\sigma_{\text {edge }}^{\mathrm{R}}(D)^{2} / \sigma_{\text {in }-2 \mathrm{D}}(D)^{2}$ and $\alpha_{2 \mathrm{D}}^{0}=\sigma_{\text {edge }}^{0}(D)^{2} / \sigma_{\text {in-2D }}(D)^{2}$. Divided $\alpha_{2 \mathrm{D}}^{\mathrm{R}}$ by $\alpha_{2 \mathrm{D}}^{0}$, it has $\alpha_{2 \mathrm{D}}^{\mathrm{R}} / \alpha_{2 \mathrm{D}}^{0}=\sigma_{\text {edge }}^{\mathrm{R}}(D)^{2} / \sigma_{\text {edge }}^{0}(D)^{2}$. Since $E_{\mathrm{c}} \propto \sigma^{-2}$, one gets $\alpha_{2 \mathrm{D}}^{\mathrm{R}}=\left[E_{\mathrm{c}}^{0}(D) / E_{\mathrm{c}}^{\mathrm{R}}(D)\right] \alpha_{2 \mathrm{D}}^{0}$, where $E_{\mathrm{c}}^{0}(D)$ and $E_{\mathrm{c}}^{\mathrm{R}}(D)$ are the respective atomic cohesive energies of edge-C atoms of naked and saturated GQDs and GNRs. Provided that $E_{\mathrm{c}}^{0}(D)$ and $E_{\mathrm{c}}^{\mathrm{R}}(D)$ have the

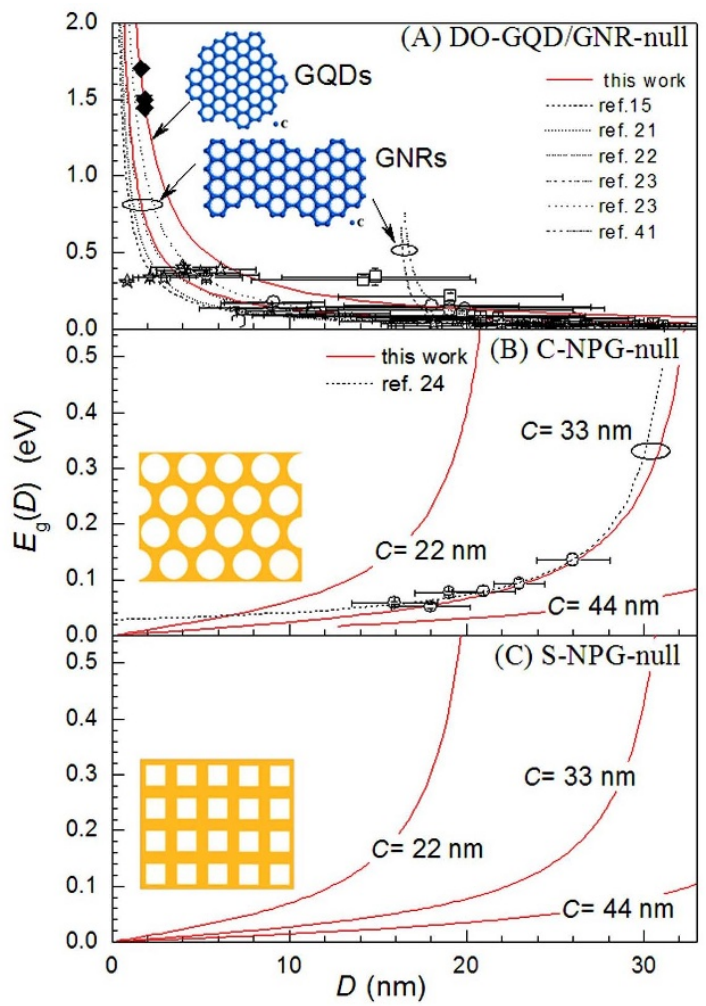

Figure $2 \mid$ The solid $E_{\mathrm{g}}(D)$ curves as the function of $D$ using equations 2.1 and 2.2 for (A) DO-GQD-null and DO-GNR-null, (B) DO-C-NPG-null and (C) DO-S-NPG-null at $C=22,33$ and $44 \mathrm{~nm}$, where $\alpha_{2 \mathrm{D}}^{0}$ and $D_{2 \mathrm{D}}^{0}$ are from Results. For the comparison purpose, the reported reciprocal or exponential fitting expressions from Table 1 are also shown using dashed and/or dotted curves with $E_{\mathrm{g}}(D)=a / D^{15,21}, E_{\mathrm{g}}(D)=a /(D-b)^{23,41}$ and $E_{\mathrm{g}}(D)=a /\left(D \mathrm{e}^{b D}\right)^{22,23}$ for GNR-null in $(\mathrm{A})$ and $E_{\mathrm{g}}(D)=a /(33-D)^{24}$ in $(\mathrm{B})$. The symbols denote available measured results with $\boldsymbol{\nabla}^{19}$ and ${ }^{20}$ for GQDs and $\square^{41}$, 岤 ${ }^{15}, \oplus^{21}, \bigotimes^{23}, \bigcirc^{3}, \Delta^{32}, \nabla^{33}$, and $\bigcirc^{34}$ for GNRs in (A) and $\bigcirc^{24}$ for C-NPGs in (B). For calculation, $h=0.142 \mathrm{~nm}^{18,35}$. $S_{\mathrm{vib}}(\infty)$ is given with $S_{\mathrm{vib}}(\infty) \approx S_{\mathrm{m}}(\infty)-R^{44,45,50}$, where $S_{\mathrm{m}}(\infty)$ means the melting entropy using $S_{\mathrm{m}}(\infty)=H_{\mathrm{m}}(\infty) / T_{\mathrm{m}}(\infty)^{44,50}$ with $H_{\mathrm{m}}(\infty)$ and $T_{\mathrm{m}}(\infty)$ being the melting enthalpy and melting point, respectively. $S_{\text {vib }}(\infty)$ or $S_{\mathrm{m}}$ of graphene can be referenced to that from graphite with $H_{\mathrm{m}}(\infty)=132 \mathrm{~kJ} / \mathrm{mol}$ and $T_{\mathrm{m}}(\infty)=5500 \mathrm{~K}^{58,59}$. same $D$-dependences, the ratio of $E_{\mathrm{c}}^{0}(D) / E_{\mathrm{c}}^{\mathrm{R}}(D)$ should be $D$ independent, and thus $\alpha_{2 \mathrm{D}}^{\mathrm{R}}=\left[E_{\mathrm{c}}^{0} / E_{\mathrm{c}}^{\mathrm{R}}\right] \alpha_{2 \mathrm{D}}^{0}$. With its help, $\alpha_{2 \mathrm{D}}^{\mathrm{R}}$ can be given when $E_{\mathrm{c}}^{0}$ and $E_{\mathrm{c}}^{\mathrm{R}}$ are known.

With the above derived expressions, Figure 3 shows the solid $E_{\mathrm{g}}(D)$ curves as the function of $D$ in light of equations 2.1 and 2.2 for DOGQD-R and DO-GNR-R with (A) $\mathrm{R}=\mathrm{H}$, (B) $\mathrm{R}=\mathrm{F}$ and (C) $\mathrm{R}=$ $\mathrm{OH}$. In comparison, the reported reciprocal fitting expression ${ }^{2}$ to depict the $D$-dependences of $E_{\mathrm{g}}(D)$ for GQD-H as aforementioned is also given using dashed curves in (A). Available experimental results (symbols) were also shown accordingly. As can be seen in Figure 3, when the edge saturation process is carried out, the BOs are realized, where $E_{\mathrm{g}}(D)$ is enhanced as $D$ decreases. There exists a fair agreement between our curves and experiment results in Figure $3 \mathrm{~A}$, which validates our theoretical models.

Investigation on electronic structure of DO-GNRs. Because of its importance in electronics, in usual, ideal AC or ZZ-GNRs have been adopted to investigate the electronic structure of graphene-based nanostructures ${ }^{13,18,29,37,52}$. However, seldom attempts have been made to it for DO-GNRs. Hence, the electronic structure of DOGNRs with the AC or ZZ skeleton along the periodic axis, referred to as DO-GNRs(AC) or DO-GNRs(ZZ), is investigated here. Figure 4 shows the band structures and the corresponding charge density isosurfaces of the lowest unoccupied molecular orbital (LUMO) and the highest occupied molecular orbital (HOMO) at $\Gamma$ point of

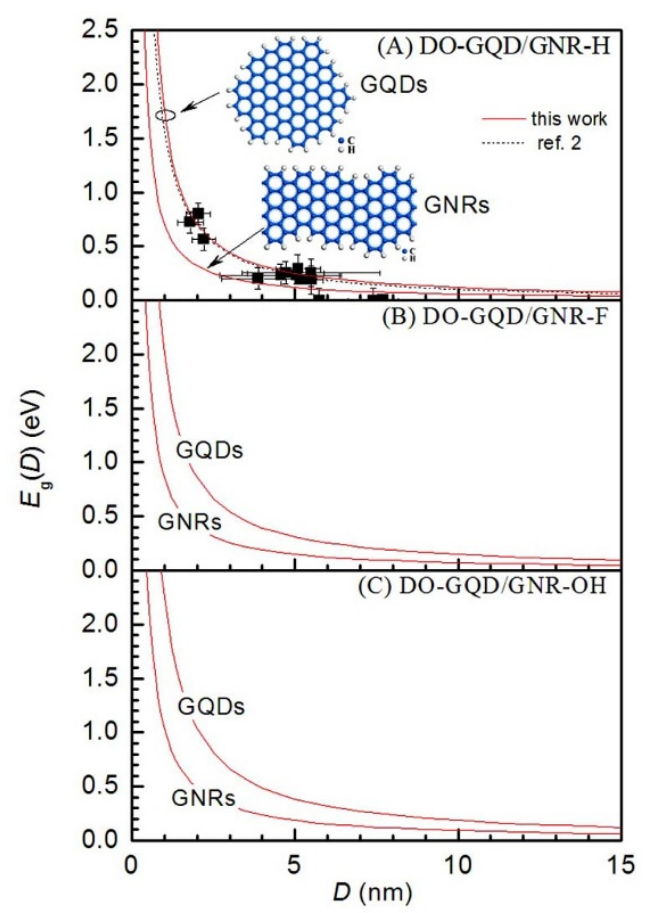

Figure $3 \mid$ The solid $E_{\mathrm{g}}(D)$ curves as the function of $D$ in light of equations 2.1 and 2.2 for DO-GQD-R and DO-GNR-R with $\mathrm{R}=\mathrm{H}$ in (A), F in (B) and $\mathrm{OH}$ in $(\mathrm{C})$, where $\alpha_{2 \mathrm{D}}^{\mathrm{R}}$ and $D_{2 \mathrm{D}}^{0}$ are from Results. In comparison, the reported reciprocal fitting expression from Table 1 to depict the $D$ dependences of $E_{\mathrm{g}}(D)$ with $E_{\mathrm{g}}(D)=a / D^{b}$ for DO-GQD-H ${ }^{2}$ is also given in (A) using dashed curve. The symbols denote the measured results with $\mathbf{\square}^{2}$. For naked GQDs or GNRs, $E_{\mathrm{c}}^{0}=E_{\mathrm{C}=\mathrm{C}}$ where $E_{\mathrm{C}=\mathrm{C}}$ is the bond energy of $\mathrm{C}$ $=\mathrm{C}$. Upon the edge saturation with $\mathrm{R}, E_{\mathrm{c}}^{\mathrm{R}}=E_{\mathrm{C}=\mathrm{C}}+E_{\mathrm{C}-\mathrm{R}} / 2 . E_{\mathrm{C}=\mathrm{C}}$ and $E_{\mathrm{C}-\mathrm{R}}$ will be taken as the averaged values from $\mathrm{ZZ}$ and AC-GNRs. The respective $E_{\mathrm{C}=\mathrm{C}}$ values are taken as 4.68 and $5.81 \mathrm{eV}$ for $E_{\mathrm{c}}^{0}$ of $\mathrm{ZZ}$ and $\mathrm{AC}$ $\mathrm{GNRs}^{56}$. To have $E_{\mathrm{c}}^{\mathrm{R}}$, the respective $E_{\mathrm{C}=\mathrm{C}}$ and $E_{\mathrm{C}-\mathrm{R}}$ are $4.97 \mathrm{eV}$ and $5.47 \mathrm{eV}$ with $\mathrm{R}=\mathrm{H}, 4.60 \mathrm{eV}$ and $5.22 \mathrm{eV}$ with $\mathrm{R}=\mathrm{F}$ and $4.44 \mathrm{eV}$ and $5.06 \mathrm{eV}$ with $\mathrm{R}$ $=\mathrm{OH}$, where the values of these parameters are given with the simulation method in Supplementary Information. See the caption of Figure 2 for other necessary parameters in calculation. 
DO-GNRs(AC) and DO-GNRs(ZZ) in (a) $\mathrm{R}=\mathrm{H}$, (b) $\mathrm{R}=\mathrm{F}$ and (c) $\mathrm{R}=\mathrm{OH}$ or of DO-GNRs(ZZ) in (d) $\mathrm{R}=\mathrm{H}$, where their width is given at $D \approx 1.2 \mathrm{~nm}$. In light of their band structures, the energy bands for spin-up and spin-down states are degenerate, while the openings are observed substantially for all DO-GNRs. The respective gaps are $0.44,0.50$ and $0.56 \mathrm{eV}$ for DO-GNRs(AC) saturated by $\mathrm{R}=$ $\mathrm{H}, \mathrm{F}$ and $\mathrm{OH}$. Regarding DO-GNRs $(\mathrm{ZZ})$ saturated with $\mathrm{R}=\mathrm{H}, E_{\mathrm{g}}(D)$ $=0.40 \mathrm{eV}$, close to that of DO-GNRs $(\mathrm{AC})$ with $\mathrm{R}=\mathrm{H}$. In comparison, some differences in HOMO and LUMO can be observed between DO-GNRs and ideal GNRs. In ideal AC-GNRs, the charge densities are concentrated homogenously along the $\mathrm{C}=\mathrm{C}$ bonds over the whole framework ${ }^{36}$. In Figure 4(a)-(c) for DOGNRs(AC), the HOMO and LUMO orbitals distribute mainly along the $\mathrm{C}=\mathrm{C}$ bonds. However, the densities are inhomogeneous over the whole region, much weak in the inner area instead. Moreover, some electronic states appear at single ZZ-structured edge-C atoms (labeled as 1, 2 and 3). In ideal ZZ-GNRs, on the other hand, the charge densities are centered at single $C$ atoms especially for those along the two $\mathrm{ZZ}$ edges ${ }^{36}$. As for DOGNRs(ZZ) in (d), however, the LUMO and HOMO charge densities are mainly centered at single $\mathrm{C}$ atoms, which seems strong in the interior area. Some charge densities along the $\mathrm{C}=\mathrm{C}$ bonds have also been observed.

\section{Discussion}

According to our predictions, the BOs observed for disordered and naked GQDs, GNRs or NPGs in Figure 2 are contributed from the decrease in $E_{\mathrm{c}}$ of $\mathrm{C}$ atoms at the edge compared to that in the interior. In a perfect graphene, each $\mathrm{C}$ atom has a planar $s p^{2}$ configuration with three $\mathrm{C}=\mathrm{C}$ bonds. When graphene flakes are naked, each edge$\mathrm{C}$ atom has one dangling bond, and it thus consists of two $\mathrm{C}=\mathrm{C}$ bonds only, leading to a decrease in $E_{c}$ of edge- $C$ atoms relative to those in the interior. Consequently, the equivalence between the $\mathrm{A}$ and $\mathrm{B}$ sublattices is broken, giving rise to the BOs in graphene flakes. As regards the saturated case in Figure 3, the dangling bonds of edge$\mathrm{C}$ atoms are terminated, while each edge- $\mathrm{C}$ atom owns two $\mathrm{C}=\mathrm{C}$ bonds and one $\mathrm{C}-\mathrm{R}$ bond, different from the case in the interior. On account of it, the equivalence of $\mathrm{A}$ and $\mathrm{B}$ atoms is broken, resulting in the BOs in GQD-R or GNR-R. This infers that the BOs in saturated GQDs and GNRs should also be ascribed to the bonding change of edge-C atoms. With regard to the difference in $E_{\mathrm{g}}(D)$ among GQDs, GNRs and NPGs, it agrees with their $D$-dependences of $l / s$ ratio, which rises for GQDs and GNRs but shrinks for NPGs on lowering $D$ (Supplementary Fig. S1). Concerning the large $E_{\mathrm{g}}(D)$ of GQDs relative to GNRs, it should be attributed to the observation that GQDs have larger $l / s$ ratios than GNRs do. It should be noted that the $D$-dependences of $E_{\mathrm{g}}(D)$ for GNR-null in Figure 2(A), NPG-null in Figure 2(B) and GNR-H in Figure 3(A) can be reflected on decreasing $D$ with those reported fitting expressions. However, these expressions can only be adopted for elucidating the BO behaviors in those graphene flakes numerically, while neither of them can be adopted to address the issues on the essential roles played by the dimension effect, geometric shape and the edge saturation, ascribed to the presence of adjustable parameters $a$ and/or $b$ in them. In
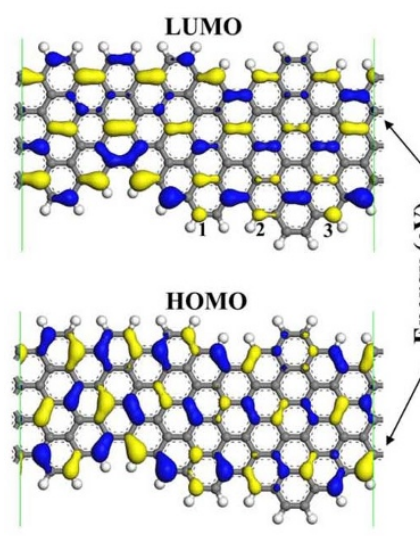

(a) $\mathbf{R}=\mathbf{H}$
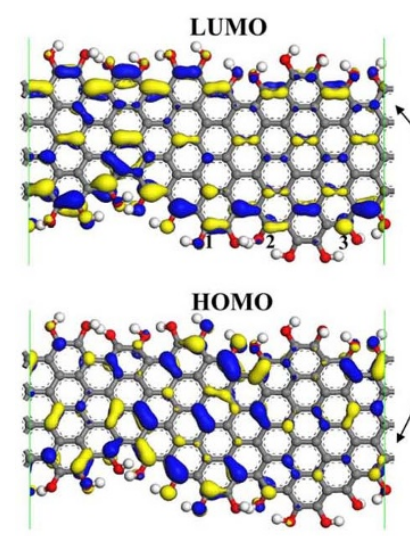

(c) $\mathrm{R}=\mathrm{OH}$
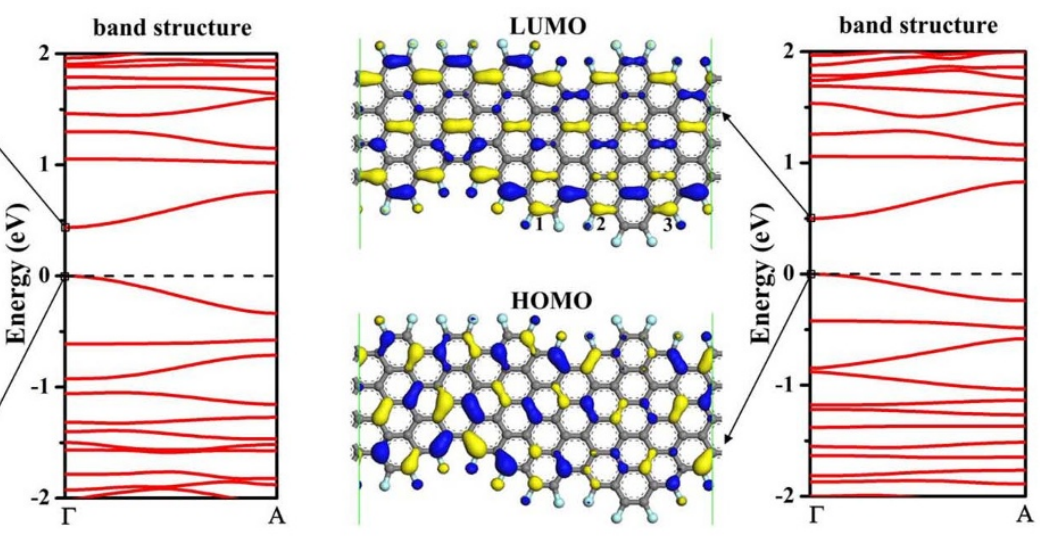

(b) $\mathbf{R}=\mathbf{F}$
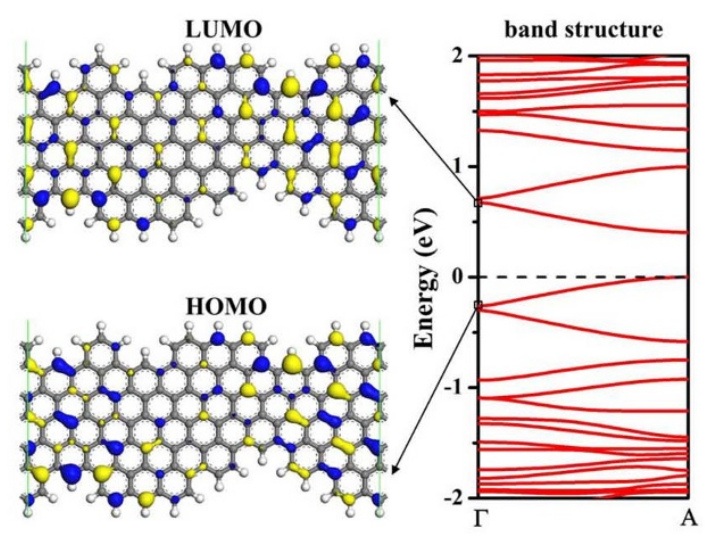

(d) $\mathbf{R}=\mathbf{H}$

Figure $4 \mid$ Band structure, charge density isosurfaces of LUMO and HOMO at $\Gamma$ point of DO-GNRs(AC) with (a) R = H, (b) R= F and (c) R= OH and of DO-GNRs(ZZ) with $(\mathbf{d}) \mathrm{R}=\mathrm{H}$. The $E_{\mathrm{F}}$ is set to zero. Blue and yellow denote the positive and negative wave function contours, respectively, and the value of the isosurfaces is $0.02 \mathrm{e} / \mathrm{A}^{3}$. C, $\mathrm{H}, \mathrm{F}$ and $\mathrm{O}$ atoms are denoted by gray, white, cyan and red balls, respectively. 
Figure 2(A), in particular, several fitting expressions have been reported to elucidate the $D$-dependence of $E_{\mathrm{g}}(D)$ for GNR-null. However, they vary considerably among different researches, and it is difficult for one to see which one is suitable for illustrating the $D$-dependence of $E_{\mathrm{g}}(D)$ for naked GNRs.

According to the previous studies, a monotonous increase of $E_{\mathrm{g}}(D)$ has been reported for ideal AC and ZZ-GQDs ${ }^{36}$, which agrees with our predictions on $E_{\mathrm{g}}(D)$ of DO-GQDs. Concerning the difference in the size of $E_{\mathrm{g}}(D)$ between AC-GQDs and ZZ-GQDs ${ }^{36}$, it should be attributed to their different edge natures ${ }^{53}$. However, the opening behaviors of disordered GNRs differ to those ideal AC and ZZGNRs. The oscillating BO behaviors in AC-GNRs are induced by the full Fermi wavelength effect ${ }^{13}$. As for ZZ-GNRs, the reported metallicity is caused by the flat bands at the Fermi level $E_{\mathrm{F}}$ from the highly localized states formed at the edges ${ }^{18}$, while the semiconducting state is stemmed from the staggered sublattice potential on the hexagonal lattice induced by the edge magnetization ${ }^{29}$. One may thus wonder whether the roles from the Fermi wavelength effect, the flat bands at $E_{\mathrm{F}}$ or the interedge magnetic interaction observed in AC or ZZ-GNRs would affect the BOs in disordered GNRs or not. Among them, in fact, the roles from the Fermi wavelength effect and the flat bands at $E_{\mathrm{F}}$ in DO-GNRs should be negligible. This is reasonable since almost no oscillating $\mathrm{BO}$ behaviors or metallic states have been reported for experimentally fabricated DO-GNRs, while the openings in them are observed substantially and rise monotonously on lowering $D$ instead $\mathrm{d}^{3,15,21,23,32-34}$. As to the role from the interedge magnetic interaction, it was investigated in the present work by performing spin-polarized and non spin-polarized calculations for DO-GNR-R shown in Figure 4(a)-(d). The obtained results show that almost no energy difference can be found for them between these two methods, indicating that all DO-GNRs give the nonmagnetic characteristics. In light of it, the contribution from the interedge magnetic interaction to the BOs in DO-GNRs should also be neglected. Obviously, the negligible roles from these factors should be attributed to the edge disorder. In Figure 4, in addition, the increasing order of $E_{\mathrm{g}}(D)$ for DO-GNR-R at $D \approx 1.2 \mathrm{~nm}$ from the simulation method with $\mathrm{R}=\mathrm{H}, \mathrm{F}$ and $\mathrm{OH}$ is the same as that from our predictions in Figure 3. However, their sizes are somewhat lower than those from our predictions, which might be related to the investigation approaches. As to the difference in the HOMO and LUMO charge density between DO-GNRs and ideal GNRs, it should also be relevant with the edge disorder in the former.

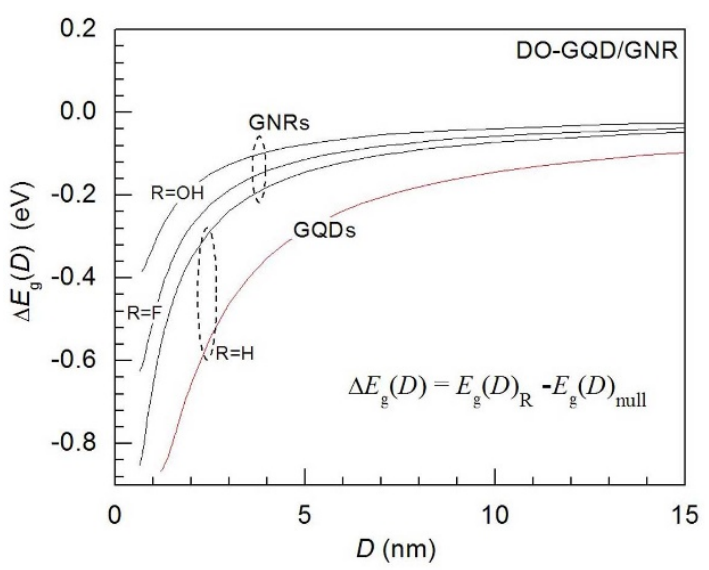

Figure $5 \mid \Delta E_{\mathrm{g}}(D)$ as the function of $D$ for disordered GQDs and GNRs between the cases with the saturated and naked edges, where $\Delta E_{\mathrm{g}}(D)=$ $E_{\mathrm{g}}(D)_{\mathrm{R}}-E_{\mathrm{g}}(D)_{\text {null }}$ with $\mathrm{R}=\mathrm{H}, \mathrm{F}$ and $\mathrm{OH}$. The corresponding values of $\alpha_{2 \mathrm{D}}^{0}, \alpha_{2 \mathrm{D}}^{\mathrm{R}}$ and $D_{2 \mathrm{D}}^{0}$ for calculation are referenced to those adopted in Figures 2 and 3 .
As mentioned above, in addition, the BOs contributed from the confinement role of $D$ of holes have been reported for some typical ideal antidot superlattices ${ }^{38-40}$, which are in agreement with our predictions on DO-NPGs. In contrast, the metallic state observed in some other superlattices should be induced by the flat bands attributed to some factors, such as the instability of the incomplete benzenoid bonding pattern, large width of the $\mathrm{ZZ}$ chains or the presence of the $\mathrm{ZZ} \mathrm{rims}{ }^{38-40}$. As the metal-semiconductor transition in porous AC-GNRs with $N_{\mathrm{a}}=11$ regarding the position effect, it is induced by the suppression of the localized states ${ }^{38}$. However, it seems that the roles from these factors can be neglected in the BOs in DO-NPGs, since they can be much suppressed by the internal edge disorder of holes. As evidence, no metallicity has been observed in experimentally fabricated DO-NPGs ${ }^{24,25,54}$. Besides it, a more recent investigation on the BOs in ideal AC or ZZ-GNRs perforated with disordered holes claims that the sensitivity of $E_{\mathrm{g}}(D)$ to the lattice symmetry is significantly suppressed in the presence of disorder ${ }^{55}$.

To see how $E_{\mathrm{g}}(D)$ of saturated DO-GQDs or GNRs will evolve relative to the naked case, Figure 5 shows $\Delta E_{\mathrm{g}}(D)$ as the function of $D$ with $\Delta E_{\mathrm{g}}(D)=E_{\mathrm{g}}(D)_{\mathrm{R}}-E_{\mathrm{g}}(D)_{\text {null }} . \Delta E_{\mathrm{g}}(D)$ are negative for the edge saturation by $\mathrm{H}, \mathrm{F}$ and $\mathrm{OH}$, decreasing on the decline of $D$. This suggests that the BOs are weakened after the edge saturation. In comparison, the $D$-dependence of $\Delta E_{\mathrm{g}}(D)$ for GQDs is stronger than that for GNRs where the $D$-dependence of $\Delta E_{\mathrm{g}}(D)$ is the strongest for the $\mathrm{H}$ saturation and the weakest for the $\mathrm{OH}$ saturation. In view of it, further, one can see that $E_{\mathrm{g}}(D)$ of GQDs or GNRs can be lined in sequence with $E_{\mathrm{g}}(D)_{\mathrm{H}}<E_{\mathrm{g}}(D)_{\mathrm{F}}<E_{\mathrm{g}}(D)_{\mathrm{OH}}<E_{\mathrm{g}}(D)_{\text {null. }}$. Frankly, such a variation is essentially relevant to the difference in $E_{\mathrm{c}}$ of edge$\mathrm{C}$ atoms for GQDs or GNRs with or without the edge saturation. Relative to naked graphene flakes, $E_{c}$ of edge- $C$ atoms is increased after the edge saturation ${ }^{56}$, depending on the radicals or groups. Accordingly, the edge physicochemical amount $\alpha_{2 \mathrm{D}}$ is suppressed, leading to the change in $E_{\mathrm{g}}(D)$ in an decreasing order with $\mathrm{R}=$ null, $\mathrm{OH}, \mathrm{F}$ and $\mathrm{H}$ as observed, Thanks to it, the opening suppression originated from the edge saturation can be understood associated with the edge physicochemical nature.

In light of equations 2.1 and 2.2, moreover, Figure 6 gives a comparison of $D$ necessary to achieve $E_{\mathrm{g}}(D)=0.1 \mathrm{eV}$ (denoted as $D_{0.1 \mathrm{eV}}$ ) and $E_{\mathrm{g}}(D)=0.4 \mathrm{eV}$ (marked with $D_{0.4 \mathrm{eV}}$ ) between naked and saturated GNRs, which are specified by $E_{\mathrm{c}}$ of edge-C atoms along the $x$ axis. From naked GNRs to saturated GNRs with $\mathrm{R}=\mathrm{OH}, \mathrm{F}$ and $\mathrm{H}$, interestingly, $D$ decreases as $E_{\mathrm{c}}$ rises, while the $D_{0.4 \mathrm{eV}}$ curve goes

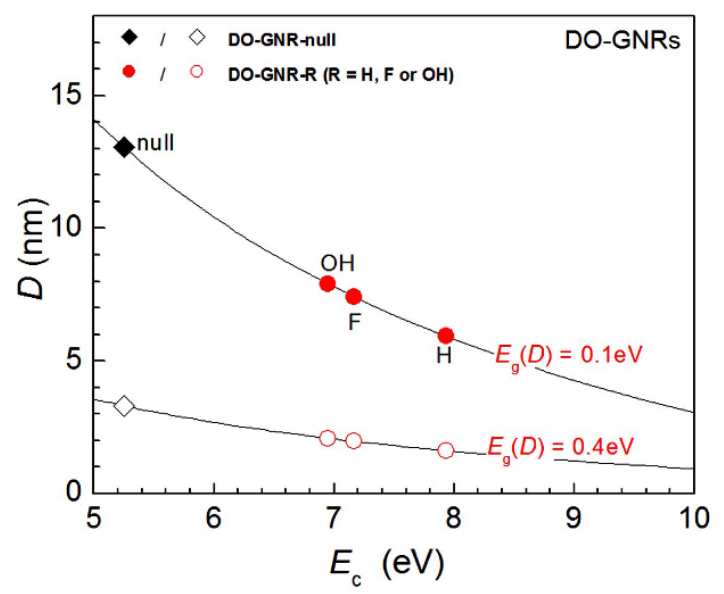

Figure $6 \mid$ A plot of $D$ necessary to achieve $E_{\mathrm{g}}(D)=0.1 \mathrm{eV}$ and $E_{\mathrm{g}}(D)=0.4 \mathrm{eV}$ for disordered GNRs with or without the edge saturation specified by $E_{\mathrm{c}}$ of edge-C atoms. The values of $E_{\mathrm{c}}^{0}, E_{\mathrm{c}}^{\mathrm{R}}, \alpha_{2 \mathrm{D}}^{0}, \alpha_{2 \mathrm{D}}^{\mathrm{R}}$ and $D_{2 \mathrm{D}}^{0}$ necessary for the calculation are referenced to those adopted in Figures 2 and 3. 


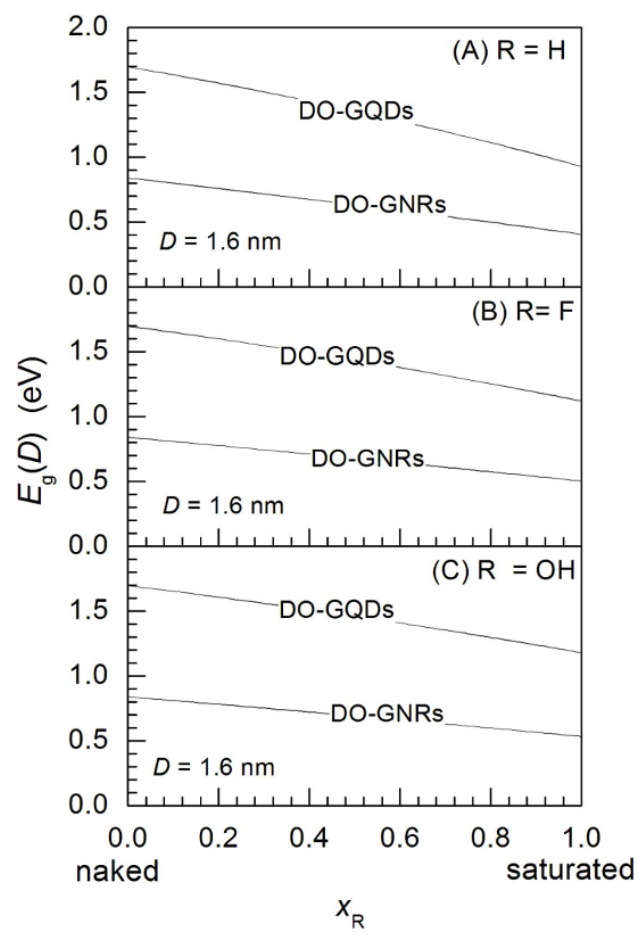

Figure $7 \mid E_{\mathrm{g}}(D)$ as the function of $x_{\mathrm{R}}$ at $D=1.6 \mathrm{~nm}$ for disordered GQDs and GNRs with $(\mathrm{A}) \mathrm{R}=\mathrm{H},(\mathrm{B}) \mathrm{R}=\mathrm{F}$ and $(\mathrm{C}) \mathrm{R}=\mathrm{OH}$ using equations 2.1 and 2.2, where the corresponding values of $E_{\mathrm{c}}^{0}, E_{\mathrm{c}}^{\mathrm{R}}, \alpha_{2 \mathrm{D}}^{0}, \alpha_{2 \mathrm{D}}^{\mathrm{R}}$ and $D_{2 \mathrm{D}}^{0}$ are referenced to those adopted in Figures 2 and 3.

below the $D_{0.1 \mathrm{eV}}$ curve. Relative to the naked case, small $D_{0.1 \mathrm{eV}}$ and $D_{0.4 \mathrm{eV}}$ are strictly required for GNR-R, decreasing in the order of $\mathrm{R}=\mathrm{OH}, \mathrm{F}$ and $\mathrm{H}$. Owing to these, one can see that, if a certain $E_{\mathrm{g}}(D)$ value is devised for a graphene flake, the necessary $D$ will be affected by the edge physicochemical nature associated with the saturation.

In published papers, special interest has been attracted how to tune the gap of graphene. One important way for it is to control the edge states or nature of graphene flakes via the edge saturation with different chemical radicals or groups ${ }^{31}$. In ZZ-GNRs, the edge modifications can break the spin degeneracy. This will lead to a semiconducting-metal transition or a half-semiconducting state with the two spin channels having different band gaps, or a spin-polarized half-semiconducting state with the spins in the valence and conduction bands being oppositely polarized. About AC-GNRs, the edge saturation gives electronic states a few $\mathrm{eV}$ away from the Fermi level and does not significantly affect their bandgap. Based on it $^{31}$, hence, it is reasonable that $E_{\mathrm{g}}(D)$ of disordered GQDs or GNRs can be modulated quantitatively by the edge saturation. In view of our predictions, since there exists an obvious discrepancy of $E_{\mathrm{g}}(D)$ between naked and saturated GQDs or GNRs, such a modulation can be realized by controlling the edge coverage upon the saturation, which can be performed through eliminating only a certain percentage of dangling bonds in a uniform way. Given that there exists a linear relationship between $\alpha_{2 \mathrm{D}}$ and the edge coverage, one has $\alpha_{2 \mathrm{D}}=\alpha_{2 \mathrm{D}}^{0}\left(1-x_{\mathrm{R}}\right)+$ $\alpha_{2 \mathrm{D}}^{\mathrm{R}} x_{\mathrm{R}}$ for partially saturated GQDs and GNRs, where $x_{\mathrm{R}}$ denotes the percentage of saturated edge- $\mathrm{C}$ atoms from 0 (the full edge-naked state) to 1 (the complete edge-saturated state). Inserting the above $\alpha_{2 \mathrm{D}}$ expression into equation $2.2, E_{\mathrm{g}}(D)$ as the function of $x_{\mathrm{R}}$ at $D=$ $1.6 \mathrm{~nm}$ was plotted in Figure 7 using equation 2.1 with $\mathrm{R}=\mathrm{H}$ in $(\mathrm{A})$, $\mathrm{R}=\mathrm{F}$ in $(\mathrm{B})$ and $\mathrm{R}=\mathrm{OH}$ in $(\mathrm{C}) . E_{\mathrm{g}}(D)$ decreases continuously as $x_{\mathrm{R}}$ rises. On increasing $x_{\mathrm{R}}$ from 0 to $1, E_{\mathrm{g}}(D)$ declines from $1.70 \mathrm{eV}$ to $0.93 \mathrm{eV}$ for GQDs and from $0.84 \mathrm{eV}$ to $0.41 \mathrm{eV}$ for GNRs in (A) with $\mathrm{R}=\mathrm{H}$, from $1.70 \mathrm{eV}$ to $1.12 \mathrm{eV}$ for GQDs and from $0.84 \mathrm{eV}$ to 0.50
$\mathrm{eV}$ for $\mathrm{GNRs}$ in (B) with $\mathrm{R}=\mathrm{F}$, and from $1.70 \mathrm{eV}$ to $1.18 \mathrm{eV}$ for GQDs and from $0.84 \mathrm{eV}$ to $0.53 \mathrm{eV}$ for GNRs in (C) with $\mathrm{R}=\mathrm{OH}$. This suggests that $E_{\mathrm{g}}(D)$ of disordered GQDs and GNRs can be adjusted subtly by changing $x_{\mathrm{R}}$, beneficial to the design and engineering of graphene-based nanoelectronic devices.

\section{Methods}

General consideration on the BOs in disordered graphene flakes. Although the dimensionality of $2 \mathrm{D}$ graphene differs from $3 \mathrm{D}$ semiconductors, there exists $\mathrm{CI}$ for edge- $\mathrm{C}$ atoms of graphene. By reason of such a similarity, the coherent energy of edge- $\mathrm{C}$ atoms will be decreased definitely. It is natural that the nearly-free-electron approach can also be adopted in predicting the BOs in graphene. In view of this, the decrease in $E_{\mathrm{c}}$ of edge- $C$ atoms will play an essential role in influencing the crystalline field of graphene, and the equivalence between the $\mathrm{A}$ and $\mathrm{B}$ sublattices will resultantly be broken, leading to the BOs in graphene.

According to the tight-binding approach ${ }^{57}$, electrons are viewed as occupying the standard orbitals of their constituent atoms, and then hopping between atoms during conduction. Mathematically, this is represented as a hopping or transfer integral between neighboring atoms, which can be viewed as the physical principle that creates electron bands in crystalline materials, due to overlapping between atomic orbitals. In virtue of it, the BOs in graphene induced by the upward shift of conduction band and downward shift of valence band of the $\pi$ bonds are associated to the hopping energy $E_{\mathrm{h}}$ between $\pi$ orbitals with $E_{\mathrm{h}}=2.96 \mathrm{eV}^{57}$. In light of these, with reference to 3D nanosemiconductors ${ }^{44,45}, E_{\mathrm{g}}(D)$ of graphene flakes is given as,

$$
E_{\mathrm{g}}(D)=\left[1-E_{\mathrm{c}}(D)_{2 \mathrm{D}} / E_{\mathrm{c}}(\infty)\right] E_{\mathrm{h}} .
$$

To obtain $E_{\mathrm{g}}(D)$ with equation $2.1, E_{\mathrm{c}}(D)_{2 \mathrm{D}} / E_{\mathrm{c}}(\infty)$ function should be explored for $2 \mathrm{D}$ flakes, which can be referenced with the $3 \mathrm{D}$ case $^{44,45,49,50}$, where free surfaces of $3 \mathrm{D}$ nanocrystals with broken bonds are substituted by free edges of $2 \mathrm{D}$ flakes. One thus has,

$$
E_{\mathrm{c}}(D)_{2 \mathrm{D}} / E_{\mathrm{c}}(\infty)=\exp \left[-\left(\alpha_{2 \mathrm{D}}-1\right) /\left(D / D_{2 \mathrm{D}}^{0}-1\right)\right] .
$$

In equation 2.2, $\alpha_{2 \mathrm{D}}$ is an edge physicochemical amount, while $D_{2 \mathrm{D}}^{0}$ denotes the critical size of $2 \mathrm{D}$ materials. To explore $E_{\mathrm{c}}(D)_{2 \mathrm{D}}$, these two amounts should be developed by considering the roles played by the dimension, geometric shape and/or the edge saturation. Referenced by the $3 \mathrm{D}$ case $\mathrm{e}^{4,45,49,50}$, the physicochemical amount $\alpha_{2 D}$ in equation 2.2 for $2 \mathrm{D}$ graphene flakes is decided by the edge nature relative to the interior case, and one thus has,

$$
\alpha_{2 \mathrm{D}}=\sigma_{\text {edge }}(D)^{2} / \sigma_{\text {in }-2 \mathrm{D}}(D)^{2},
$$

where the subscripts edge and in-2D mean the edge and interior atoms of $2 \mathrm{D}$ graphene flakes. In principle, $\alpha_{2 D}$ of naked graphene flakes marked with $\alpha_{2 \mathrm{D}}^{0}$ is decided by the CI of $\mathrm{C}$ edge-atoms. Since the $\mathrm{CI}$ of edge-C atoms is eliminated upon the edge saturation, in contrast, a different $\alpha_{2 \mathrm{D}}$ will be resulted from it for saturated graphene flakes denoted with $\alpha_{2 \mathrm{D}}^{\mathrm{R}}$. On the other hand, $D_{2 \mathrm{D}}^{0}$ is one amount related to the dimensionality or the geometrical shape of graphene flakes, which can be principally given as,

$$
D / D_{2 D}^{0}=s / l h
$$

where $s / l$ is the area/edge ratio. Associated to it, the $D$-dependences of $E_{\mathrm{g}}(D)$ should be relevant to the $l / s$ ratio of graphene flakes.

DFT approach to electronic structure of DO-GNRs. The electronic structure of DO-GNRs was investigated with the DFT approach. DO-GNRs are $1.2 \mathrm{~nm}$ wide or so with the AC or ZZ skeleton along the periodic axis, referred to as DO-GNRs(AC) or DO-GNRs(ZZ). Moreover, the two edges of both DO-GNRs(AC) and DO-

GNRs(ZZ) are composed of the mixed AC or ZZ structures and kept symmetric. For DO-GNRs(AC), the supercell includes 12 zigzag $\mathrm{C}=\mathrm{C}$ bonds in the periodic direction, as shown in Figure 4(a)-(c), where the edges are saturated by $\mathrm{H}, \mathrm{F}$ and $\mathrm{OH}$, respectively. For DO-GNRs(ZZ), the supercell has 12 armchair $\mathrm{C}=\mathrm{C}$ bonds in the periodic direction [see Figure 4(d)], where the edge is saturated by $\mathrm{H}$ atoms.

Spin-polarized DFT calculations are performed using DMOL3 code with GGA$\mathrm{PBE}$ as the exchange correlation function. All electron core treatment and double numeric plus polarization (DNP) basis set are adopted. The Brillouin zone is sampled by $5 \times 1 \times 1 \mathrm{k}$-points and the real space global cutoff radius is set to be $5.0 \AA$ for the geometry and electronic calculations. In addition, a uniform vacuum of $15 \AA$ perpendicular to the GNR is employed to ensure no interactions between neighboring cells. These bring out the convergence tolerance of energy of $1 \times 10^{-5} \mathrm{Ha}$, maximum force of $0.002 \mathrm{Ha}^{-1}$ and maximum displacement of $0.005 \AA$, respectively.

1. Xue, J. M. et al. Long-wavelength local density of states oscillations near graphene step edges. Phys. Rev. Lett. 108 (2012).

2. Ritter, K. A. \& Lyding, J. W. The influence of edge structure on the electronic properties of graphene quantum dots and nanoribbons. Nat. Mater. 8, 235-242 (2009).

3. Lu, Y. et al. High-On/Off-Ratio Graphene Nanoconstriction Field-Effect Transistor. Small 6, 2748-2754 (2010).

4. Vicarelli, L. et al. Graphene field-effect transistors as room-temperature terahertz detectors. Nat. Mater. 11, 865-871 (2012). 
5. Titov, A. V., Kral, P. \& Pearson, R. Sandwiched Graphene-Membrane Superstructures. ACS Nano 4, 229-234 (2010).

6. Nguyen, P. \& Berry, V. Graphene Interfaced with Biological Cells: Opportunities and Challenges. J. Phys. Chem. lett. 3, 1024-1029 (2012).

7. Mohanty, N. \& Berry, V. Graphene-Based Single-Bacterium Resolution Biodevice and DNA Transistor: Interfacing Graphene Derivatives with Nanoscale and Microscale Biocomponents. Nano Lett. 8, 4469-4476 (2008).

8. Hong, X. et al. Unusual resistance hysteresis in n-layer graphene field effect transistors fabricated on ferroelectric $\mathrm{Pb}(\mathrm{Zr} 0.2 \mathrm{Ti0}$.8)O-3. Appl. Phys. Lett. 97 (2010).

9. DaSilva, A. M., Zou, K., Jain, J. K. \& Zhu, J. Mechanism for Current Saturation and Energy Dissipation in Graphene Transistors. Phys. Rev. Lett. 104 (2010).

10. Engel, M. et al. Light-matter interaction in a microcavity-controlled graphene transistor. Nat Commun 3 (2012).

11. Yankowitz, M. et al. Emergence of superlattice Dirac points in graphene on hexagonal boron nitride. Nat. Phys. 8, 382-386 (2012).

12. Sun, C. Q., Fu, S. Y. \& Nie, Y. G. Dominance of Broken Bonds and Unpaired Nonbonding pi-Electrons in the Band Gap Expansion and Edge States Generation in Graphene Nanoribbons. J. Phys. Chem. C 112, 18927-18934 (2008)

13. Barone, V., Hod, O. \& Scuseria, G. E. Electronic structure and stability of semiconducting graphene nanoribbons. Nano Lett. 6, 2748-2754 (2006).

14. Soldano, C., Mahmood, A. \& Dujardin, E. Production, properties and potential of graphene. Carbon 48, 2127-2150 (2010).

15. Li, X. et al. Chemically Derived, Ultrasmooth Graphene Nanoribbon Semiconductors. Science 319, 1229-1232 (2008)

16. Wang, X. et al. Room-Temperature All-Semiconducting Sub-10-nm Graphene Nanoribbon Field-Effect Transistors. Phys. Rev. Lett. 100, 206803 (2008).

17. Ihn, T. et al. Graphene single-electron transistors. Mater. Today 13, 44-50 (2010).

18. Nakada, K., Fujita, M., Dresselhaus, G. \& Dresselhaus, M. S. Edge state in graphene ribbons: Nanometer size effect and edge shape dependence. Phys. Rev. B 54, 17954-17961 (1996).

19. Yan, X., Cui, X., Li, B. S. \& Li, L. S. Large, Solution-Processable Graphene Quantum Dots as Light Absorbers for Photovoltaics. Nano Lett. 10, 1869-1873 (2010).

20. Yan, X., Cui, X. \& Li, L. S. Synthesis of Large, Stable Colloidal Graphene Quantum Dots with Tunable Size. J. Am. Chem. Soc. 132, 5944 (2010).

21. Bai, J. W., Duan, X. F. \& Huang, Y. Rational Fabrication of Graphene Nanoribbons Using a Nanowire Etch Mask. Nano Lett. 9, 2083-2087 (2009).

22. Sols, F., Guinea, F. \& Neto, A. H. C. Coulomb Blockade in Graphene Nanoribbons. Phys. Rev. Lett. 99, 166803 (2007).

23. Molitor, F. et al. Energy and transport gaps in etched graphene nanoribbons. Semicond. Sci. Technol. 25, 034002 (2010).

24. Liang, X. et al. Formation of Bandgap and Subbands in Graphene Nanomeshes with Sub-10 nm Ribbon Width Fabricated via Nanoimprint Lithography. Nano Lett. 10, 2454-2460 (2010).

25. Bai, J. et al. Graphene nanomesh. Nat. Nanotechnol. 5, 190-194 (2010).

26. Liu, X., Oostinga, J. B., Morpurgo, A. F. \& Vandersypen, L. M. K. Electrostatic confinement of electrons in graphene nanoribbons. Phys. Rev. B 80, 121407 (2009)

27. Todd, K., Chou, H.-T., Amasha, S. \& Goldhaber-Gordon, D. Quantum Dot Behavior in Graphene Nanoconstrictions. Nano Lett. 9, 416-421 (2008).

28. Yoon, Y. \& Guo, J. Effect of edge roughness in graphene nanoribbon transistors. Appl. Phys. Lett. 91, 073103 (2007).

29. Son, Y.-W., Cohen, M. L. \& Louie, S. G. Energy Gaps in Graphene Nanoribbons. Phys. Rev. Lett. 97, 216803 (2006).

30. Kudin, K. N. Zigzag graphene nanoribbons with saturated edges. ACS Nano 2, 516-522 (2008).

31. Cervantes-Sodi, F., Csanyi, G., Piscanec, S. \& Ferrari, A. C. Edge-functionalized and substitutionally doped graphene nanoribbons: Electronic and spin properties. Phys. Rev. B 77, 165427 (2008).

32. Wang, X. R. \& Dai, H. J. Etching and narrowing of graphene from the edges. Nat. Chem. 2, 661-665 (2010)

33. Chen, Z. H., Lin, Y. M., Rooks, M. J. \& Avouris, P. Graphene nano-ribbon electronics. Physica E 40, 228-232 (2007).

34. Jiao, L. Y. et al. Narrow graphene nanoribbons from carbon nanotubes. Nature 458, 877-880 (2009).

35. Zhang, T., Xue, Q. Z., Zhang, S. \& Dong, M. D. Theoretical approaches to graphene and graphene-based materials. Nano Today 7, 180-200 (2012).

36. Silva, A. M. et al. Graphene Nanoflakes: Thermal Stability, Infrared Signatures, and Potential Applications in the Field of Spintronics and Optical Nanodevices. J. Phys. Chem. C 114, 17472-17485 (2010).

37. Ezawa, M. Peculiar width dependence of the electronic properties of carbon nanoribbons. Phys. Rev. B 73, 045432 (2006).
38. Baskin, A. \& Kral, P. Electronic structures of porous nanocarbons. Sci. Rep. 1, 36 (2011).

39. Ouyang, F. P. et al. Electronic Structure and Chemical Modification of Graphene Antidot Lattices. J. Phys. Chem. C 114, 15578-15583 (2010).

40. Petersen, R., Pedersen, T. G. \& Jauho, A. P. Clar Sextet Analysis of Triangular, Rectangular, and Honeycomb Graphene Antidot Lattices. ACS Nano 5, 523-529 (2011).

41. Han, M. Y., Ozyilmaz, B., Zhang, Y. B. \& Kim, P. Energy Band-Gap Engineering of Graphene Nanoribbons. Phys. Rev. Lett. 98, 206805 (2007).

42. Gleiter, H. Nanostructured materials: basic concepts and microstructure. Acta Mater. 48, 1-29 (2000).

43. Sun, C. Thermo-mechanical behavior of low-dimensional systems: The local bond average approach. Prog. Mater. Sci. 54, 179-307 (2009).

44. Yang, C. \& Jiang, Q. Size effect on the bandgap of II-VI semiconductor nanocrystals. Mater. Sci. Eng. B 131, 191-194 (2006).

45. Zhu, Y. F., Lang, X. Y. \& Jiang, Q. The Effect of Alloying on the Bandgap Energy of Nanoscaled Semiconductor Alloys. Adv. Funct. Mater. 18, 1422-1429 (2008).

46. Li, S. \& Yang, G. W. Universal scaling of semiconductor nanowires bandgap. Appl. Phys. Lett. 95 (2009).

47. Jiang, Q. \& Lu, H. M. Size dependent interface energy and its applications. Surf. Sci. Rep. 63, 427-464 (2008).

48. Ouyang, G., Wang, C. X. \& Yang, G. W. Surface Energy of Nanostructural Materials with Negative Curvature and Related Size Effects. Chem. Rev. 109, 4221-4247 (2009).

49. Jiang, Q., Shi, H. X. \& Zhao, M. Melting thermodynamics of organic nanocrystals. J. Chem. Phys. 111, 2176-2180 (1999).

50. Jiang, Q., Zhang, S. \& Zhao, M. Size-dependent melting point of noble metals. Mater. Chem. Phys. 82, 225-227 (2003).

51. Acik, M. \& Chabal, Y. J. Nature of Graphene Edges: A Review. Jpn. J. Appl. Phys. 50, 070101 (2011).

52. Rochefort, A., Salahub, D. R. \& Avouris, P. Effects of Finite Length on the Electronic Structure of Carbon Nanotubes. J. Phys. Chem. B 103, 641-646 (1999).

53. Ouyang, G., Sun, C. Q. \& Zhu, W. G. Atomistic Origin and Pressure Dependence of Band Gap Variation in Semiconductor Nanocrystals. J. Phys. Chem. C 113 , 9516-9519 (2009).

54. Kim, M. et al. Fabrication and Characterization of Large-Area, Semiconducting Nanoperforated Graphene Materials. Nano Lett. 10, 1125-1131 (2010).

55. Nguyen, V. H., Nguyen, M. C., Nguyen, H.-V. \& Dollfus, P. Disorder effects on electronic bandgap and transport in graphene-nanomesh-based structures. J. Appl. Phys. 113, 013702-013709 (2013).

56. Koskinen, P., Malola, S. \& Häkkinen, H. Self-Passivating Edge Reconstructions of Graphene. Phys. Rev. Lett. 101, 115502 (2008).

57. Reich, S., Maultzsch, J., Thomsen, C. \& Ordejon, P. Tight-binding description of graphene. Phys. Rev. B 66, 035412 (2002).

58. Bundy, F. P. Melting of Graphite at Very High Pressure. J. Chem. Phys. 38, 618-630 (1963)

59. Korobenko, V. N., Savvatimski, A. I. \& Cheret, R. Graphite Melting and Properties of Liquid Carbon. Int. J. Thermophys. 20, 1247-1256 (1999).

\section{Acknowledgements}

Financial supports are acknowledged by National Key Basic Research Development Program (Grant No. 2010CB631001)

\section{Author contributions}

Y. F. Z. established the theoretical predictions, plotted all the figures and wrote the paper. Q. Q. D. and M. Z. conducted the computer simulation. Q. J. designed the project and guided the research. Y. F. Z. and Q. J. revised the paper. All authors contributed to the discussions.

\section{Additional information}

Supplementary information accompanies this paper at http://www.nature.com/ scientificreports

Competing financial interests: The authors declare no competing financial interests.

License: This work is licensed under a Creative Commons

Attribution-NonCommercial-NoDerivs 3.0 Unported License. To view a copy of this license, visit http://creativecommons.org/licenses/by-nc-nd/3.0/

How to cite this article: Zhu, Y.F., Dai, Q.Q., Zhao, M. \& Jiang, Q. Physicochemical insight into gap openings in graphene. Sci. Rep. 3, 1524; DOI:10.1038/srep01524 (2013). 